\title{
PERIODIC AND NONNEGATIVE PERIODIC SOLUTIONS OF NONLINEAR NEUTRAL DYNAMIC EQUATIONS ON A TIME SCALE
}

\section{MANEL GOUASMIA ${ }^{1}$, ABDELOUAHEB ARDJOUNI ${ }^{1,2, *}$ AND AHCENE DJOUDI ${ }^{1}$}

${ }^{1}$ Applied Mathematics Lab, Faculty of Sciences, Department of Mathematics, Univ Annaba, P.O. Box 12, Annaba 23000, Algeria

${ }^{2}$ Faculty of Sciences and Technology, Department of Mathematics and Informatics, Univ Souk Ahras, P.O. Box 1553, Souk Ahras, 41000, Algeria

*Corresponding author: abd_ardjouni@yahoo.fr

\begin{abstract}
Let $\mathbb{T}$ be a periodic time scale. We use Krasnoselskii-Burton's fixed point theorem to show new results on the existence of periodic and nonnegative periodic solutions of nonlinear neutral dynamic equation with variable delay of the form

$$
x^{\Delta}(t)=-a(t) h\left(x^{\sigma}(t)\right)+Q(t, x(t-\tau(t)))^{\Delta}+G(t, x(t), x(t-\tau(t))), t \in \mathbb{T} .
$$

We invert the given equation to obtain an equivalent integral equation from which we define a fixed point mapping written as a sum of a large contraction and a completely continuous map. The Caratheodory condition is used for the functions $Q$ and $G$. The results obtained here extend the work of Mesmouli, Ardjouni and Djoudi [16].
\end{abstract}

\section{Introduction}

In 1988, Stephan Hilger [11] introduced the theory of time scales (measure chains) as a means of unifying discrete and continuum calculi. Since Hilger's initial work there has been significant growth in the theory

2010 Mathematics Subject Classification. 34A37, 34A12, 35B09, 35B10, 45J05.

Key words and phrases. Krasnoselskii-Burton's theorem; large contraction; neutral differential equation; integral equation; periodic solution; time scales.

(C)2018 Authors retain the copyrights of their papers, and all open access articles are distributed under the terms of the Creative Commons Attribution License. 
of dynamic equations on time scales, covering a variety of different problems; see $[8,9,14]$ and references therein.

Let $\mathbb{T}$ be a periodic time scale such that $0 \in \mathbb{T}$. In this article, we are interested in the analysis of qualitative theory of periodic and positive periodic solutions of neutral dynamic equations. Motivated by the papers [1]- [7], [10], [12], [13], [15], [16] and the references therein, we consider the following nonlinear neutral dynamic equation

$$
x^{\Delta}(t)=-a(t) h\left(x^{\sigma}(t)\right)+Q(t, x(t-\tau(t)))^{\Delta}+G(t, x(t), x(t-\tau(t))), t \in \mathbb{T} .
$$

Throughout this paper we assume that $a$ and $\tau$ are positive rd-continuous functions, $i d-\tau: \mathbb{T} \rightarrow \mathbb{T}$ is increasing so that the function $x(t-\tau(t))$ is well defined over $\mathbb{T}$. The function $h$ is continuous, $Q$ and $G$ satisfying the Caratheodory condition. To reach our desired end we have to transform (1.1) into an integral equation written as a sum of two mapping, one is a contraction and the other is continuous and compact. After that, we use Krasnoselskii-Burton's fixed point theorem, to show the existence of periodic and nonnegative periodic solutions.

The organization of this paper is as follows. In Section 2, we introduce some notations and definitions, and state some preliminary material needed in later sections. We will state some facts about the exponential function on a time scale as well as the fixed point theorems. For details on fixed point theorems we refer the reader to $[10,17]$. In Section 3, we establish the existence of periodic solutions. In Section 4, we give sufficient conditions to ensure the existence of nonnegative periodic solutions. The results presented in this paper extend the main results in $[16]$.

\section{Preliminaries}

In this section, we consider some advanced topics in the theory of dynamic equations on a time scales. Again, we remind that for a review of this topic we direct the reader to the monographs of Bohner and Peterson [8] and [9].

A time scale $\mathbb{T}$ is a closed nonempty subset of $\mathbb{R}$. For $t \in \mathbb{T}$ the forward jump operator $\sigma$, and the backward jump operator $\rho$, respectively, are defined as $\sigma(t)=\inf \{s \in \mathbb{T}: s>t\}$ and $\rho(t)=\sup \{s \in \mathbb{T}: s<t\}$. These operators allow elements in the time scale to be classified as follows. We say $t$ is right scattered if $\sigma(t)>t$ and right dense if $\sigma(t)=t$. We say $t$ is left scattered if $\rho(t)<t$ and left dense if $\rho(t)=t$. The graininess function $\mu: \mathbb{T} \rightarrow[0, \infty)$, is defined by $\mu(t)=\sigma(t)-t$ and gives the distance between an element and its successor. We set $\inf \varnothing=\sup \mathbb{T}$ and $\sup \varnothing=\inf \mathbb{T}$. If $\mathbb{T}$ has a left scattered maximum $M$, we define $\mathbb{T}^{k}=\mathbb{T} \backslash\{M\}$. Otherwise, we define $\mathbb{T}^{k}=\mathbb{T}$. If $\mathbb{T}$ has a right scattered minimum $m$, we define $\mathbb{T}^{k}=\mathbb{T} \backslash\{m\}$. Otherwise, we define $\mathbb{T}^{k}=\mathbb{T}$. 
Let $t \in \mathbb{T}^{k}$ and let $f: \mathbb{T} \rightarrow \mathbb{R}$. The delta derivative of $f(t)$, denoted $f^{\Delta}(t)$, is defined to be the number (when it exists), with the property that, for each $\varepsilon>0$, there is a neighborhood $U$ of $t$ such that

$$
\left|f(\sigma(t))-f(s)-f^{\Delta}(t)[\sigma(t)-s]\right| \leq \varepsilon|\sigma(t)-s|,
$$

for all $s \in U$. If $\mathbb{T}=\mathbb{R}$ then $f^{\Delta}(t)=f^{\prime}(t)$ is the usual derivative. If $\mathbb{T}=\mathbb{Z}$ then $f^{\Delta}(t)=\Delta f(t)=$ $f(t+1)-f(t)$ is the forward difference of $f$ at $t$.

A function is right dense continuous (rd-continuous), $f \in C_{r d}=C_{r d}(\mathbb{T}, \mathbb{R})$, if it is continuous at every right dense point $t \in \mathbb{T}$ and its left-hand limits exist at each left dense point $t \in \mathbb{T}$. The function $f: \mathbb{T} \rightarrow \mathbb{R}$ is differentiable on $\mathbb{T}^{k}$ provided $f^{\Delta}(t)$ exists for all $t \in \mathbb{T}^{k}$.

We are now ready to state some properties of the delta-derivative of $f$. Note $f^{\sigma}(t)=f(\sigma(t))$.

Theorem 2.1 ( $\left[8\right.$, Theorem 1.20]). Assume $f, g: \mathbb{T} \rightarrow \mathbb{R}$ are differentiable at $t \in \mathbb{T}^{k}$ and let $\alpha$ be a scalar.

(i) $(f+g)^{\Delta}(t)=f^{\Delta}(t)+g^{\Delta}(t)$.

(ii) $(\alpha f)^{\Delta}(t)=\alpha f^{\Delta}(t)$.

(iii) The product rules

$$
\begin{aligned}
& (f g)^{\Delta}(t)=f^{\Delta}(t) g(t)+f^{\sigma}(t) g^{\Delta}(t), \\
& (f g)^{\Delta}(t)=f(t) g^{\Delta}(t)+f^{\Delta}(t) g^{\sigma}(t) .
\end{aligned}
$$

(iv) If $g(t) g^{\sigma}(t) \neq 0$ then

$$
\left(\frac{f}{g}\right)^{\Delta}(t)=\frac{f^{\Delta}(t) g(t)-f(t) g^{\Delta}(t)}{g(t) g^{\sigma}(t)}
$$

Definition 2.1 ( [12]). We say that a time scale $\mathbb{T}$ is periodic if there exist a $w>0$ such that if $t \in \mathbb{T}$ then $t \pm w \in \mathbb{T}$. For $\mathbb{T} \neq \mathbb{R}$, the smallest positive $w$ is called the period of the time scale.

Definition $2.2([12])$. Let $\mathbb{T} \neq \mathbb{R}$ be a periodic time scales with the period $w$. We say that the function $f: \mathbb{T} \rightarrow \mathbb{R}$ is periodic with period $T$ if there exists a natural number $n$ such that $T=n w, f(t \pm T)=f(t)$ for all $t \in \mathbb{T}$ and $T$ is the smallest number such that $f(t \pm T)=f(t)$. If $\mathbb{T}=\mathbb{R}$, we say that $f$ is periodic with period $T>0$ if $T$ is the smallest positive number such that $f(t \pm T)=f(t)$ for all $t \in \mathbb{T}$.

Remark 2.1 ( [12]). If $\mathbb{T}$ is a periodic time scale with period $w$, then $\sigma(t \pm n w)=\sigma(t) \pm n w$. Consequently, the graininess function $\mu$ satisfies $\mu(t \pm n w)=\sigma(t \pm n w)-(t \pm n w)=\sigma(t)-t=\mu(t)$ and so, is a periodic function with period $w$.

The next theorem is the chain rule on time scales ( [8, Theorem 1.93]).

Theorem 2.2 (Chain Rule). Assume $v: \mathbb{T} \rightarrow \mathbb{R}$ is strictly increasing and $\widetilde{\mathbb{T}}:=v(\mathbb{T})$ is a time scale. Let $w: \widetilde{\mathbb{T}} \rightarrow \mathbb{R}$. If $v^{\Delta}(t)$ and $w^{\widetilde{\Delta}}(v(t))$ exist for $t \in \mathbb{T}^{k}$, then

$$
(w \circ v)^{\Delta}=\left(w^{\widetilde{\Delta}} \circ v\right) v^{\Delta} .
$$


In the sequel we will need to differentiate and integrate functions of the form $f(t-\tau(t))=f(v(t))$ where, $v(t):=t-\tau(t)$. Our next theorem is the substitution rule ( $[8$, Theorem 1.98]).

Theorem 2.3. Assume $v: \mathbb{T} \rightarrow \mathbb{R}$ is strictly increasing and $\widetilde{\mathbb{T}}:=v(\mathbb{T})$ is a time scale. If $f: \mathbb{T} \rightarrow \mathbb{R}$ is rd-continuous function and $v$ is differentiable with rd-continuous derivative, then for $a, b \in T$,

$$
\int_{a}^{b} f(t) v^{\Delta}(t) \Delta t=\int_{v(a)}^{v(b)}\left(f \circ v^{-1}\right)(s) \widetilde{\Delta} s .
$$

A function $p: \mathbb{T} \rightarrow \mathbb{R}$ is said to be regressive provided $1+\mu(t) p(t) \neq 0$ for all $t \in \mathbb{T}^{k}$. The set of all regressive rd-continuous function $f: \mathbb{T} \rightarrow \mathbb{R}$ is denoted by $\mathcal{R}$. The set of all positively regressive functions $\mathcal{R}^{+}$, is given by $\mathcal{R}^{+}=\{f \in \mathcal{R}: 1+\mu(t) f(t)>0$ for all $t \in \mathbb{T}\}$.

Let $p \in \mathcal{R}$ and $\mu(t) \neq 0$ for all $t \in \mathbb{T}$. The exponential function on $\mathbb{T}$ is defined by

$$
e_{p}(t, s)=\exp \left(\int_{s}^{t} \frac{1}{\mu(z)} \log (1+\mu(z) p(z)) \Delta z\right) .
$$

It is well known that if $p \in \mathcal{R}^{+}$, then $e_{p}(t, s)>0$ for all $t \in \mathbb{T}$. Also, the exponential function $y(t)=e_{p}(t, s)$ is the solution to the initial value problem $y^{\Delta}=p(t) y, y(s)=1$. Other properties of the exponential function are given by the following lemma.

Lemma 2.1 ( $[8$, Theorem 2.36]). Let $p, q \in \mathcal{R}$. Then

(i) $e_{0}(t, s)=1$ and $e_{p}(t, t)=1$,

(ii) $e_{p}(\sigma(t), s)=(1+\mu(t) p(t)) e_{p}(t, s)$,

(iii) $\frac{1}{e_{p}(t, s)}=e_{\ominus p}(t, s)$, where $e_{\ominus p}(t, s)=-\frac{p(t)}{1+\mu(t) p(t)}$,

(iv) $e_{p}(t, s)=\frac{1}{e_{p}(s, t)}=e_{\ominus p}(t, s)$,

(v) $e_{p}(t, s) e_{p}(s, r)=e_{p}(t, r)$,

(vi) $e_{p}^{\Delta}(., s)=p e_{p}(., s)$ and $\left(\frac{1}{e_{p}(., s)}\right)^{\Delta}=-\frac{p(t)}{e_{p}^{\sigma}(., s)}$.

Theorem 2.4 ( $\left[7\right.$, Theorem 2.1]). Let $\mathbb{T}$ be a periodic time scale with period $w>0$. If $p \in C_{r d}(\mathbb{T})$ is a periodic function with the period $T=n w$, then

$$
\int_{a+T}^{b+T} p(u) \Delta u=\int_{a}^{b} p(u) \Delta u, e_{p}(b+T, a+T)=e_{p}(b, a) \text { if } p \in \mathcal{R},
$$

and $e_{p}(t+T, t)$ is independent of $t \in \mathbb{T}$ whenever $p \in \mathcal{R}$.

Lemma 2.2 ( [1]). If $p \in \mathcal{R}^{+}$, then

$$
0<e_{p}(t, s) \leq \exp \left(\int_{s}^{t} p(u) \Delta u\right), \forall t \in \mathbb{T} .
$$

Corollary 2.1 ( [1]). If $p \in \mathcal{R}^{+}$and $p(t)<0$ for all $t \in \mathbb{T}$, then for all $s \in \mathbb{T}$ with $s \leq t$ we have

$$
0<e_{p}(t, s) \leq \exp \left(\int_{s}^{t} p(u) \Delta u\right)<1 \text {. }
$$


Now, we give some definitions which we are going to use in what follows.

Definition 2.3. A function $f:[0, T] \times \mathbb{R}^{n} \rightarrow \mathbb{R}$ is an $L_{\Delta}^{1}$-Caratheodory function if it satisfies the following conditions

(i) For each $z \in \mathbb{R}^{n}$, the mapping $t \mapsto f(t, z)$ is $\Delta$-measurable.

(ii) For almost all $t \in[0, T]$, the mapping $z \mapsto f(t, z)$ is continuous on $\mathbb{R}^{n}$.

(iii) For each $r>0$, there exists $\alpha_{r} \in L_{\Delta}^{1}([0, T], \mathbb{R})$ such that for almost all $t \in[0, T]$ and for all $z$ such that $|z|<r$, we have $|f(t, z)| \leq \alpha_{r}(t)$.

Burton observed that Krasnoselskii's result (see [17]) can be more attractive in applications with certain changes and formulated Theorem 2.5 below (see [10] for the proof).

Definition 2.4. Let $(\mathcal{M}, d)$ be a metric space and assume that $B: \mathcal{M} \mapsto \mathcal{M}$. $B$ is said to be a large contraction, if for $\varphi, \psi \in \mathcal{M}$, with $\varphi \neq \psi$, we have $d(B \varphi, B \psi)<d(\varphi, \psi)$, and if $\forall \varepsilon>0, \exists \delta<1$ such that

$$
[\varphi, \psi \in \mathcal{M}, d(\varphi, \psi) \geq \varepsilon] \Rightarrow d(B \varphi, B \psi)<\delta d(\varphi, \psi)
$$

It is proved in [10] that a large contraction defined on a closed bounded and complete metric space has a unique fixed point.

Theorem 2.5 (Krasnoselskii-Burton). Let $\mathcal{M}$ be a closed bounded convex nonempty subset of a Banach space $(\mathcal{B},\|\|$.$) . Suppose that A$ and $B$ map $\mathcal{M}$ into $\mathcal{M}$ such that

(i) A is completely continuous,

(ii) B is large contraction,

(iii) $x, y \in \mathcal{M}$, implies $A x+B y \in \mathcal{M}$.

Then there exists $z \in \mathcal{M}$ with $z=A z+B z$.

\section{Existence OF PERIODIC SOLUtions}

Let $T>0, T \in \mathbb{T}$ be fixed and if $\mathbb{T} \neq \mathbb{R}, T=n w$ for some $n \in \mathbb{N}$. By the notation $[a, b]$ we mean $[a, b]=\{t \in \mathbb{T}: a \leq t \leq b\}$, unless otherwise specified. The intervals $[a, b),(a, b]$ and $(a, b)$ are defined similarly. Define

$$
P_{T}=\{\phi \in C(\mathbb{T}, \mathbb{R}), \phi(t+T)=\phi(t)\}
$$

where $C(\mathbb{T}, \mathbb{R})$ is the space of all real valued rd-continuous functions. Then $\left(P_{T},\|\cdot\|\right)$ is a Banach space when it is endowed with the supremum norm

$$
\|\phi\|=\sup _{t \in[0, T]}|\phi(t)| .
$$

We will need the following lemma whose proof can be found in [12]. 
Lemma 3.1. Let $x \in P_{T}$. Then $\left\|x^{\sigma}\right\|=\|x \circ \sigma\|$ exists and $\left\|x^{\sigma}\right\|=\|x\|$.

In this paper we assume that $h$ is continuous, $a \in \mathcal{R}^{+}$is rd-continuous and

$$
a(t-T)=a(t), \tau(t-T)=\tau(t), \tau(t) \geq \tau^{*}>0,
$$

with $\tau$ continuously and $\tau^{*}$ is positive constant, $a$ is positive function and

$$
1-e_{\ominus a}(t, t-T) \equiv \frac{1}{\eta} \neq 0
$$

The functions $Q(t, x)$ and $G(t, x, y)$ are periodic in $t$ of period $T$. That is

$$
Q(t-T, x)=Q(t, x), G(t-T, x, y)=G(t, x, y)
$$

The following lemma is fundamental to our results.

Lemma 3.2. Suppose (3.1)-(3.3) hold. If $x \in P_{T}$, then $x$ is a solution of equation (1.1) if and only if

$$
\begin{aligned}
x(t) & =\eta \int_{t-T}^{t} k(t, u) a(u)\left[x^{\sigma}(u)-h\left(x^{\sigma}(u)\right)\right] \Delta u+Q(t, x(t-\tau(t))) \\
& +\eta \int_{t-T}^{t} k(t, u)\left[-a(u) Q^{\sigma}(u, x(u-\tau(u)))+G(u, x(u), x(u-\tau(u)))\right] \Delta u,
\end{aligned}
$$

where

$$
k(t, u)=e_{\ominus a}(t, u)
$$

Proof. Let $x \in P_{T}$ be a solution of (1.1). Rewrite the equation (1.1) as

$$
\begin{aligned}
& (x(t)-Q(t, x(t-\tau(t))))^{\Delta}+a(t)\left[x^{\sigma}(t)-Q^{\sigma}(t, x(t-\tau(t)))\right] \\
& =a(t)\left[x^{\sigma}(t)-h\left(x^{\sigma}(t)\right)\right]-a(t) Q^{\sigma}(t, x(t-\tau(t)))+G(t, x(t), x(t-\tau(t))) .
\end{aligned}
$$

Multiply both sides of the above equation by $e_{a}(t, 0)$ and then integrate from $t-T$ to $t$ to obtain

$$
\begin{aligned}
& \int_{t-T}^{t}\left[(x(u)-Q(u, x(u-\tau(u)))) e_{a}(u, 0)\right]^{\Delta} \Delta u \\
& =\int_{t-T}^{t} a(u)\left[x^{\sigma}(u)-h\left(x^{\sigma}(u)\right)\right] e_{a}(u, 0) \Delta u \\
& +\int_{t-T}^{t}\left[-a(u) Q^{\sigma}(u, x(u-\tau(u)))+G(u, x(u), x(u-\tau(u)))\right] e_{a}(u, 0) \Delta u .
\end{aligned}
$$


As a consequence, we arrive at

$$
\begin{aligned}
& (x(t)-Q(t, x(t-\tau(t)))) e_{a}(t, 0) \\
& -(x(t-T)-Q(t-T, x(t-T-\tau(t-T)))) e_{a}(t-T, 0) \\
& =\int_{t-T}^{t} a(u)\left[x^{\sigma}(u)-h\left(x^{\sigma}(u)\right)\right] e_{a}(u, 0) \Delta u \\
& +\int_{t-T}^{t}\left[-a(u) Q^{\sigma}(u, x(u-\tau(u)))+G(u, x(u), x(u-\tau(u)))\right] e_{a}(u, 0) \Delta u .
\end{aligned}
$$

By dividing both sides of the above equation by $e_{a}(t, 0)$ and using the fact that $x(t)=x(t-T)$, we obtain

$$
\begin{aligned}
& x(t)-Q(t, x(t-\tau(t))) \\
& =\eta \int_{t-T}^{t} a(u)\left[x^{\sigma}(u)-h\left(x^{\sigma}(u)\right)\right] e_{\ominus a}(t, u) \Delta u \\
& +\eta \int_{t-T}^{t}\left[-a(u) Q^{\sigma}(u, x(u-\tau(u)))+G(u, x(u), x(u-\tau(u)))\right] e_{\ominus a}(t, u) \Delta u .
\end{aligned}
$$

The converse implication is easily obtained and the proof is complete.

To apply Theorem 2.5, we need to define a Banach space $\mathcal{B}$, a closed bounded convex subset $\mathcal{M}$ of $\mathcal{B}$ and construct two mappings; one is a completely continuous and the other is large contraction. So, we let $(\mathcal{B},\|\cdot\|)=\left(P_{T},\|\cdot\|\right)$ and

$$
\mathcal{M}=\left\{\varphi \in P_{T},\|\varphi\| \leq L\right\}
$$

with $L \in(0,1]$. For $x \in \mathcal{M}$, let the mapping $H$ be defined by

$$
H(x)=x^{\sigma}-h\left(x^{\sigma}\right)
$$

and by (3.4), define the mapping $S: P_{T} \rightarrow P_{T}$ by

$$
\begin{aligned}
& (S \varphi)(t) \\
& =\eta \int_{t-T}^{t} k(t, u) a(u) H(\varphi(u)) \Delta u+Q(t, \varphi(t-\tau(t))) \\
& +\eta \int_{t-T}^{t} k(t, u)\left[-a(u) Q^{\sigma}(u, \varphi(u-\tau(u)))+G(u, \varphi(u), \varphi(u-\tau(u)))\right] \Delta u .
\end{aligned}
$$

Therefore, we express the above equation as

$$
(S \varphi)(t)=(A \varphi)(t)+(B \varphi)(t)
$$


where $A, B: P_{T} \rightarrow P_{T}$ are given by

$$
\begin{aligned}
& (A \varphi)(t) \\
& =Q(t, \varphi(t-\tau(t))) \\
& +\eta \int_{t-T}^{t} k(t, u)\left[-a(u) Q^{\sigma}(u, \varphi(u-\tau(u)))+G(u, \varphi(u), \varphi(u-\tau(u)))\right] \Delta u .
\end{aligned}
$$

and

$$
(B \varphi)(t)=\eta \int_{t-T}^{t} k(t, u) a(u) H(\varphi(u)) \Delta u .
$$

We will assume that the following conditions hold.

(H1) $a \in L_{\Delta}^{1}[0, T]$ is bounded.

(H2) $Q$ and $G$ satisfy Caratheodory conditions with respect to $L_{\Delta}^{1}[0, T]$.

(H3) There exist periodic functions $q_{1}, q_{2} \in L_{\Delta}^{1}[0, T]$, with period $T$, such that

$$
|Q(t, x)| \leq q_{1}(t)|x|+q_{2}(t)
$$

(H4) There exist periodic functions $g_{1}, g_{2}, g_{3} \in L_{\Delta}^{1}[0, T]$, with period $T$, such that

$$
|G(t, x, y)| \leq g_{1}(t)|x|+g_{2}(t)|y|+g_{3}(t)
$$

Now, we need the following assumptions

$$
\begin{gathered}
q_{1}(t) L+q_{2}(t) \leq \frac{\gamma_{1}}{2} L, \\
g_{1}(t) L+g_{2}(t) L+g_{3}(t) \leq \gamma_{2} L a(t),
\end{gathered}
$$

and

$$
J\left(\gamma_{1}+\gamma_{2}\right) \leq 1
$$

where $\gamma_{1}, \gamma_{2}$ and $J$ are positive constants with $J \geq 3$.

Lemma 3.3. For $A$ defined in (3.10), suppose that (3.1)-(3.3), (3.12)-(3.14) and (H1)-(H4) hold. Then $A: \mathcal{M} \rightarrow \mathcal{M}$.

Proof. Let $A$ be defined by (3.10). Obviously, $A \varphi$ is rd-continuous. First by (3.1) and (3.3), a change of variable in (3.10) shows that $(A \varphi)(t+T)=(A \varphi)(t)$. That is, if $\varphi \in P_{T}$ then $A \varphi$ is periodic with period $T$. 
Next, let $\varphi \in \mathcal{M}$, by (3.12)-(3.14) and (H1)-(H4) we have

$$
\begin{aligned}
& |(A \varphi)(t)| \\
& \leq|Q(t, \varphi(t-\tau(t)))| \\
& +\eta \int_{t-T}^{t} k(t, u)\left[a(u)\left|Q^{\sigma}(u, \varphi(u-\tau(u)))\right|+|G(u, \varphi(u), \varphi(u-\tau(u)))|\right] \Delta u \\
& \leq q_{1}(t)|\varphi(t-\tau(t))|+q_{2}(t) \\
& +\eta \int_{t-T}^{t} k(t, u) a(u)\left[q_{1}(u)|\varphi(u-\tau(u))|+q_{2}(u)\right] \Delta u \\
& +\eta \int_{t-T}^{t} k(t, u)\left[g_{1}(u)|\varphi(u)|+g_{2}(u)|\varphi(u-\tau(u))|+g_{3}(u)\right] \Delta u \\
& \leq \gamma_{1} L+\gamma_{2} L \leq \frac{L}{J} \leq L .
\end{aligned}
$$

That is $A \varphi \in \mathcal{M}$.

Lemma 3.4. For $A: \mathcal{M} \rightarrow \mathcal{M}$ defined in (3.10), suppose that (3.1)-(3.3), (3.12)-(3.14) and (H1)-(H4) hold. Then $A$ is completely continuous.

Proof. We show that $A$ is continuous in the supremum norm, Let $\varphi_{n} \in \mathcal{M}$ where $n$ is a positive integer such that $\varphi_{n} \rightarrow \varphi$ as $n \rightarrow \infty$. Then

$$
\begin{aligned}
& \left|\left(A \varphi_{n}\right)(t)-(A \varphi)(t)\right| \\
& \leq \mid Q\left(t, \varphi_{n}(t-\tau(t))-Q(t, \varphi(t-\tau(t)) \mid\right. \\
& +\eta \int_{t-T}^{t} k(t, u) a(u)\left|Q^{\sigma}\left(u, \varphi_{n}(u-\tau(u))\right)-Q^{\sigma}(u, \varphi(u-\tau(u)))\right| \Delta u \\
& +\eta \int_{t-T}^{t} k(t, u)\left|G\left(u, \varphi_{n}(u), \varphi_{n}(u-\tau(u))\right)-G(u, \varphi(u), \varphi(u-\tau(u)))\right| \Delta u .
\end{aligned}
$$

By the Dominated Convergence Theorem, $\lim _{n \rightarrow \infty}\left|\left(A \varphi_{n}\right)(t)-(A \varphi)(t)\right|=0$. Then $A$ is continuous.

We next show that $A$ is completely continuous. Let $\varphi \in \mathcal{M}$, then, by Lemma 3.3, we see that

$$
\|A \varphi\| \leq L
$$


And so the family of functions $A \varphi$ is uniformly bounded. Again, let $\varphi \in \mathcal{M}$. Without loss of generality, we can pick $\omega<t$ such that $t-\omega<T$. Then

$$
\begin{aligned}
& |(A \varphi)(t)-(A \varphi)(\omega)| \\
& \leq|Q(t, \varphi(t-\tau(t)))-Q(\omega, \varphi(\omega-\tau(\omega)))| \\
& +\eta \mid \int_{t-T}^{t} k(t, u) a(u) Q^{\sigma}(u, \varphi(u-\tau(u))) \Delta u \\
& -\int_{\omega-T}^{\omega} k(\omega, u) a(u) Q^{\sigma}(u, \varphi(u-\tau(u))) \Delta u \mid \\
& +\eta \mid \int_{t-T}^{t} k(t, u) G(u, \varphi(u), \varphi(u-\tau(u))) \Delta u \\
& -\int_{\omega-T}^{\omega} k(\omega, u) G(u, \varphi(u), \varphi(u-\tau(u))) \Delta u \mid \\
& \leq|Q(t, \varphi(t-\tau(t)))-Q(\omega, \varphi(\omega-\tau(\omega)))| \\
& +2 \eta k_{0} \int_{\omega-T}^{t-T}\left[a(u) q_{L}(u)+g_{\sqrt{2} L}(u)\right] \Delta u \\
& +\eta \int_{\omega-T}^{\omega}|k(t, u)-k(\omega, u)|\left[a(u) q_{L}(u)+g_{\sqrt{2} L}(u)\right] \Delta u \\
& \leq|Q(t, \varphi(t-\tau(t)))-Q(\omega, \varphi(\omega-\tau(\omega)))| \\
& +2 \eta k_{0} \int_{\omega}^{t}\left[a(u) q_{L}(u)+g_{\sqrt{2} L}(u)\right] \Delta u \\
& +\eta \int_{0}^{T}|k(t, u)-k(\omega, u)|\left[a(u) q_{L}(u)+g_{\sqrt{2} L}(u)\right] \Delta u
\end{aligned}
$$

where $k_{0}=\max _{u \in[t-T, t]}\{k(t, u)\}$, then by the Dominated Convergence Theorem $|(A \varphi)(t)-(A \varphi)(\omega)| \rightarrow 0$ as $t-\omega \rightarrow 0$ independently of $\varphi \in \mathcal{M}$. Thus $(A \varphi)$ is equicontinuous. Hence by Ascoli-Arzela's theorem $A$ is completely continuous.

Now, we state an important result see [1] and for convenience we present below its proof, we deduce by this theorem that the following are sufficient conditions implying that the mapping $H$ given by (3.8) is a large contraction on the set $\mathcal{M}$.

(H5) $h: \mathbb{R} \rightarrow \mathbb{R}$ is continuous on $[-L, L]$ and differentiable on $(-L, L)$,

(H6) the function $h$ is strictly increasing on $[-L, L]$,

(H7) $\sup _{t \in(-L, L)} h^{\prime}(t) \leq 1$.

Theorem 3.1. Let $h: \mathbb{R} \rightarrow \mathbb{R}$ be a function satisfying (H5)-(H7). Then the mapping $H$ in (3.8) is a large contraction on the set $\mathcal{M}$. 
Proof. Let $\varphi^{\sigma}, \psi^{\sigma} \in \mathcal{M}$ with $\varphi^{\sigma} \neq \psi^{\sigma}$. Then $\varphi^{\sigma}(t) \neq \psi^{\sigma}(t)$ for some $t \in \mathbb{T}$. Let us denote the set of all such $t$ by $D(\varphi, \psi)$, i.e.,

$$
D(\varphi, \psi)=\left\{t \in \mathbb{T}: \varphi^{\sigma}(t) \neq \psi^{\sigma}(t)\right\}
$$

For all $t \in D(\varphi, \psi)$, we have

$$
\begin{aligned}
& |(H \varphi)(t)-(H \psi)(t)| \\
& \leq\left|\varphi^{\sigma}(t)-\psi^{\sigma}(t)-h\left(\varphi^{\sigma}(t)\right)+h\left(\psi^{\sigma}(t)\right)\right| \\
& \leq\left|\varphi^{\sigma}(t)-\psi^{\sigma}(t)\right|\left|1-\frac{h\left(\varphi^{\sigma}(t)\right)-h\left(\psi^{\sigma}(t)\right)}{\varphi^{\sigma}(t)-\psi^{\sigma}(t)}\right| .
\end{aligned}
$$

Since $h$ is a strictly increasing function we have

$$
\frac{h\left(\varphi^{\sigma}(t)\right)-h\left(\psi^{\sigma}(t)\right)}{\varphi^{\sigma}(t)-\psi^{\sigma}(t)}>0 \text { for all } t \in D(\varphi, \psi) .
$$

For each fixed $t \in D(\varphi, \psi)$ define the interval $I_{t} \subset[-L, L]$ by

$$
I_{t}=\left\{\begin{array}{l}
\left(\varphi^{\sigma}(t), \psi^{\sigma}(t)\right) \text { if } \varphi^{\sigma}(t)<\psi^{\sigma}(t), \\
\left(\psi^{\sigma}(t), \varphi^{\sigma}(t)\right) \text { if } \psi^{\sigma}(t)<\varphi^{\sigma}(t) .
\end{array}\right.
$$

The Mean Value Theorem implies that for each fixed $t \in D(\varphi, \psi)$ there exists a real number $c_{t} \in I_{t}$ such that

$$
\frac{h\left(\varphi^{\sigma}(t)\right)-h\left(\psi^{\sigma}(t)\right)}{\varphi^{\sigma}(t)-\psi^{\sigma}(t)}=h^{\prime}\left(c_{t}\right)
$$

By (H6) and (H7) we have

$$
0 \leq \inf _{u \in(-L, L)} h^{\prime}(u) \leq \inf _{u \in I_{t}} h^{\prime}(u) \leq h^{\prime}\left(c_{t}\right) \leq \sup _{u \in I_{t}} h^{\prime}(u) \leq \sup _{u \in(-L, L)} h^{\prime}(u) \leq 1 .
$$

Hence, by (3.15)-(3.17) we obtain

$$
|(H \varphi)(t)-(H \psi)(t)| \leq\left|\varphi^{\sigma}(t)-\psi^{\sigma}(t)\right|\left|1-\inf _{u \in(-L, L)} h^{\prime}(u)\right|,
$$

for all $t \in D(\varphi, \psi)$. This implies a large contraction in the supremum norm. To see this, choose a fixed $\varepsilon \in(0,1)$ and assume that $\varphi$ and $\psi$ are two functions in $\mathcal{M}$ satisfying

$$
\varepsilon \leq \sup _{t \in(-L, L)}|\varphi(t)-\psi(t)|=\|\varphi-\psi\| .
$$

If $\left|\varphi^{\sigma}(t)-\psi^{\sigma}(t)\right| \leq \frac{\varepsilon}{2}$ for some $t \in D(\varphi, \psi)$, then we get by (3.17) and (3.18) that

$$
|(H \varphi)(t)-(H \psi)(t)| \leq\left|\varphi^{\sigma}(t)-\psi^{\sigma}(t)\right| \leq \frac{1}{2}\|\varphi-\psi\| .
$$

Since $h$ is continuous and strictly increasing, the function $h\left(u+\frac{\varepsilon}{2}\right)-h(u)$ attains its minimum on the closed and bounded interval $[-L, L]$. Thus, if $\frac{\varepsilon}{2} \leq\left|\varphi^{\sigma}(t)-\psi^{\sigma}(t)\right|$ for some $t \in D(\varphi, \psi)$, then by (H6) and (H7) we conclude that

$$
1 \geq \frac{h\left(\varphi^{\sigma}(t)\right)-h\left(\psi^{\sigma}(t)\right)}{\varphi^{\sigma}(t)-\psi^{\sigma}(t)}>\lambda
$$


where

$$
\lambda:=\frac{1}{2 L} \min \left\{h\left(u+\frac{\varepsilon}{2}\right)-h(u): u \in[-L, L]\right\}>0 .
$$

Hence, (3.15) implies

$$
|(H \varphi)(t)-(H \psi)(t)| \leq(1-\lambda)\|\varphi-\psi\| .
$$

Consequently, combining (3.19) and (3.20) we obtain

$$
|(H \varphi)(t)-(H \psi)(t)| \leq \delta\|\varphi-\psi\|,
$$

where

$$
\delta=\max \left\{\frac{1}{2}, 1-\lambda\right\}
$$

The proof is complete.

The next result shows the relationship between the mappings $H$ and $B$ in the sense of large contractions. Assume that

$$
\max \{|H(-L)|,|H(L)|\} \leq \frac{2 L}{J} .
$$

Lemma 3.5. Let $B$ be defined by (3.11), suppose (H5)-(H7) hold. Then B: $\mathcal{M} \rightarrow \mathcal{M}$ is a large contraction.

Proof. Let $B$ be defined by (3.11). Obviously, $B \varphi$ is continuous and it is easy to show that $(B \varphi)(t+T)=$ $(B \varphi)(t)$. Let $\varphi \in \mathcal{M}$

$$
\begin{aligned}
|(B \varphi)(t)| & \leq \eta \int_{t-T}^{t} k(t, u) a(u) \max \{|H(-L)|,|H(L)|\} \Delta u \\
& \leq \frac{2 L}{J}<L
\end{aligned}
$$

which implies $B: \mathcal{M} \rightarrow \mathcal{M}$.

By Theorem 3.1, $H$ is large contraction on $\mathcal{M}$, then for any $\varphi, \psi \in \mathcal{M}$, with $\varphi \neq \psi$ and for any $\varepsilon>0$, from the proof of that Theorem, we have found a $\delta<1$, such that

$$
\begin{aligned}
|(B \varphi)(t)-(B \psi)(t)| & =\left|\eta \int_{t-T}^{t} k(t, u) a(u)[H(\varphi(u))-H(\psi(u))] \Delta u\right| \\
& \leq \delta\|\varphi-\psi\| \eta \int_{t-T}^{t} k(t, u) a(u) \Delta u \leq \delta\|\varphi-\psi\| .
\end{aligned}
$$

The proof is complete.

Theorem 3.2. Suppose the hypothesis of Lemmas 3.3, 3.4 and 3.5 hold. Let $\mathcal{M}$ defined by (3.7). Then the equation (1.1) has a T-periodic solution in $\mathcal{M}$. 
Proof. By Lemma 3.3, 3.4, $A$ is continuous and $A(\mathcal{M})$ is contained in a compact set. Also, from Lemma 3.5 , the mapping $B$ is a large contraction. Next, we show that if $\varphi, \psi \in \mathcal{M}$, we have $\|A \psi+B \varphi\| \leq L$. Let $\varphi, \psi \in \mathcal{M}$ with $\|\varphi\|,\|\psi\| \leq L$. By (3.12)-(3.14)

$$
\begin{aligned}
\|A \psi+B \varphi\| & \leq\left(\gamma_{1}+\gamma_{2}\right) L+\frac{2 L}{J} \\
& \leq \frac{L}{J}+\frac{2 L}{J} \leq L .
\end{aligned}
$$

Clearly, all the hypotheses of the Krasnoselskii-Burton's theorem are satisfied. Thus there exists a fixed point $z \in \mathcal{M}$ such that $z=A z+B z$. By Lemma 3.2 this fixed point is a solution of (1.1). Hence (1.1) has a $T$-periodic solution.

\section{EXISTENCE OF NONNEGATIVE PERIODIC SOlUtions}

In this section we obtain the existence of a nonnegative periodic solution of (1.1). By applying Theorem 2.5 , we need to define a closed, convex, and bounded subset $\mathbb{M}$ of $P_{T}$. So, let

$$
\mathbb{M}=\left\{\phi \in P_{T}: 0 \leq \phi \leq K\right\},
$$

where $K$ is positive constant. To simplify notation, we let

$$
m=\min _{u \in[t-T, t]} e_{\ominus a}(t, u), \quad M=\max _{u \in[t-T, t]} e_{\ominus a}(t, u) .
$$

It is easy to see that for all $(t, u) \in[0,2 T]^{2}$,

$$
m \leq k(t, u) \leq M \text {. }
$$

Then we obtain the existence of a nonnegative periodic solution of (1.1) by considering the two cases;

1) $Q(t, y) \geq 0, \forall t \in[0, T], y \in \mathbb{M}$.

2) $Q(t, y) \leq 0, \forall t \in[0, T], y \in \mathbb{M}$.

In the case one, we assume for all $t \in[0, T], x, y \in \mathbb{M}$, that there exists a positive constant $c_{1}$ such that

$$
\begin{gathered}
0 \leq Q(t, y) \leq c_{1} y, \\
c_{1}<1, \\
0 \leq-a(t) Q^{\sigma}(t, y)+G(t, x, y) \\
a(t) H(\varphi(t))-a(t) Q^{\sigma}(t, y)+G(t, x, y) \leq \frac{K\left(1-c_{1}\right)}{M \eta T} .
\end{gathered}
$$

Lemma 4.1. Let $A$ and $B$ given by (3.10) and (3.11) respectively, assume that (4.4)-(4.7) hold. Then $A, B: \mathbb{M} \rightarrow \mathbb{M}$. 
Proof. Let $A$ defined by (3.11). So, for any $\varphi \in \mathbb{M}$, we have

$$
\begin{aligned}
0 & \leq(A \varphi)(t) \leq Q(t, \varphi(t-\tau(t))) \\
& +\eta \int_{t-T}^{t} k(t, u)\left[-a(u) Q^{\sigma}(u, \varphi(u-\tau(u)))+G(u, \varphi(u), \varphi(u-\tau(u)))\right] \Delta u \\
& \leq \eta \int_{t-T}^{t} M \frac{K\left(1-c_{1}\right)}{M \eta T} \Delta u+c_{1} K=K,
\end{aligned}
$$

That is $A \varphi \in \mathbb{M}$.

Now, let $B$ defined by (3.11). So, for any $\varphi \in \mathbb{M}$, we have

$$
0 \leq(B \varphi)(t) \leq \eta \int_{t-T}^{t} M \frac{K\left(1-c_{1}\right)}{M \eta T} \Delta u \leq M \eta T \frac{K}{M \eta T}=K
$$

That is $B \varphi \in \mathbb{M}$.

Theorem 4.1. Suppose the hypothesis of Lemmas 3.4, 3.5 and 4.1 hold. Then equation (1.1) has a nonnegative $T$-periodic solution $x$ in the subset $\mathbb{M}$.

Proof. By Lemma 3.4, $A$ is completely continuous. Also, from Lemma 3.5, the mapping $B$ is a large contraction. By Lemma 4.1, $A, B: \mathbb{M} \rightarrow \mathbb{M}$. Next, we show that if $\varphi, \psi \in \mathbb{M}$, we have $0 \leq A \psi+B \varphi \leq K$. Let $\varphi, \psi \in \mathbb{M}$ with $0 \leq \varphi, \psi \leq K$. By (4.4)-(4.7)

$$
\begin{aligned}
& (A \psi)(t)+(B \varphi)(t) \\
& =\eta \int_{t-T}^{t} k(t, u) a(u) H(\varphi(u)) \Delta u+Q(t, \psi(t-\tau(t))) \\
& +\eta \int_{t-T}^{t} k(t, u)\left[-a(u) Q^{\sigma}(u, \psi(u-\tau(u)))+G(u, \psi(u), \psi(u-\tau(u)))\right] \Delta u \\
& \leq \eta \int_{t-T}^{t} k(t, u) \frac{K\left(1-c_{1}\right)}{M \eta T} \Delta u+c_{1} K \\
& \leq \eta \int_{t-T}^{t} M \frac{K\left(1-c_{1}\right)}{M \eta T} \Delta u+c_{1} K=K
\end{aligned}
$$

On the other hand,

$$
(A \psi)(t)+(B \varphi)(t) \geq 0 .
$$

Clearly, all the hypotheses of the Krasnoselskii-Burton's theorem are satisfied. Thus there exists a fixed point $z \in \mathbb{M}$ such that $z=A z+B z$. By Lemma 1 this fixed point is a solution of (1.1) and the proof is complete.

In the case two, we substitute conditions (4.4)-(4.7) with the following conditions respectively. We assume that there exist a negative constant $c_{2}$ such that

$$
c_{2} y \leq Q(t, y) \leq 0
$$




$$
\begin{gathered}
-c_{2}<1, \\
\frac{-c_{2} K}{M \eta T} \leq a(t) H(\varphi(t))-a(t) Q(t, y)+G(t, x, y), \\
a(t) H(\varphi(t))-a(t) Q(t, y)+G(t, x, y) \leq \frac{K}{M \eta T} .
\end{gathered}
$$

Theorem 4.2. Suppose (4.8)-(4.11) and the hypothesis of Lemmas 3.3, 3.4 and 3.5 hold. Then equation (1.1) has a nonnegative $T$-periodic solution $x$ in the subset $\mathbb{M}$.

Proof. By Lemma 3.3, 3.4, $A$ is completely continuous. Also, from Lemma 3.5, the mapping $B$ is a large contraction. To see that, it is easy to show as in Lemma 4.1, $A, B: \mathbb{M} \rightarrow \mathbb{M}$. Next, we show that if $\varphi, \psi \in \mathbb{M}$, we have $0 \leq A \psi+B \varphi \leq K$. Let $\varphi, \psi \in \mathbb{M}$, with $0 \leq \varphi, \psi \leq K$. By (4.8)- (4.11)

$$
\begin{aligned}
& (A \psi)(t)+(B \varphi)(t) \\
& =\eta \int_{t-T}^{t} k(t, u) a(u) H(\varphi(u)) \Delta u+Q(t, \psi(t-\tau(t))) \\
& +\eta \int_{t-T}^{t} k(t, u)\left[-a(u) Q^{\sigma}(u, \psi(u-\tau(u)))+G(u, \psi(u), \psi(u-\tau(u)))\right] \Delta u \\
& \leq \eta \int_{t-T}^{t} k(t, u) \frac{K}{M \eta T} \Delta u=\eta \int_{t-T}^{t} M \frac{K}{M \eta T} \Delta u=K .
\end{aligned}
$$

On the other hand,

$$
(A \psi)(t)+(B \varphi)(t) \geq \eta \int_{t-T}^{t} M \frac{-c_{2} K}{M \eta T} \Delta u+c_{2} K=0 .
$$

Clearly, all the hypotheses of the Krasnoselskii-Burton's theorem are satisfied. Thus there exists a fixed point $z \in \mathbb{M}$ such that $z=A z+B z$. By Lemma 3.2 this fixed point is a solution of (1.1) and the proof is complete.

\section{REFERENCES}

[1] M. Adivar and Y. N. Raffoul, Existence of periodic solutions in totally nonlinear delay dynamic equations, Electronic Journal of Qualitative Theory of Differential Equations, 2009, No. 1, 1-20.

[2] A. Ardjouni and A. Djoudi, Existence of periodic solutions for nonlinear neutral dynamic equations with variable delay on a time scale. Commun. Nonlinear Sci. Numer. Simulat., 17 (2012), 3061-3069.

[3] A. Ardjouni and A. Djoudi, Existence of positive periodic solutions for nonlinear neutral dynamic equations with variable delay on a time scale, Malaya J. Mat. 2(1) (2013) 60-67.

[4] A. Ardjouni and A. Djoudi, Existence of periodic solutions for nonlinear neutral dynamic equations with functional delay on a time scale, Acta Univ. Palacki. Olomnc., Fac. rer. nat., Mathematica 52, 1 (2013) 5-19.

[5] A. Ardjouni and A. Djoudi, A. Existence, uniqueness and positivity of solutions for a neutral nonlinear periodic dynamic equation on a time scale, J. Nonlinear Anal. Optim. 6 (2) (2015), 19-29.

[6] M. Belaid, A. Ardjouni and A.Djoudi, Stability in totally nonlinear neutral dynamic equations on time scales, Int. J. Anal. Appl. 11 (2) (2016), 110-123. 
[7] L. Bi, M. Bohner and M. Fan, Periodic solutions of functional dynamic equations with infinite delay, Nonlinear Anal. 68 (2008), 1226-1245.

[8] M. Bohner, A. Peterson, Dynamic Equations on Time Scales, An Introduction with Applications, Birkhäuser, Boston, 2001.

[9] M. Bohner, A. Peterson, Advances in Dynamic Equations on Time Scales, Birkhäuser, Boston, 2003.

[10] T. A. Burton, Stability by Fixed Point Theory for Functional Differential Equations, Dover Publications, New York, 2006.

[11] S. Hilger, Ein Masskettenkalkül mit Anwendung auf Zentrumsmanningfaltigkeiten. PhD thesis, Universität Würzburg, 1988.

[12] E. R. Kaufmann and Y. N. Raffoul, Periodic solutions for a neutral nonlinear dynamical equation on a time scale, J. Math. Anal. Appl. 319 (2006), no. 1, 315-325.

[13] E. R. Kaufmann and Y. N. Raffoul, Periodicity and stability in neutral nonlinear dynamic equations with functional delay on a time scale, Electron. J. Differential Equations, 2007 (2007), No. 27, 1-12.

[14] V. Lakshmikantham, S. Sivasundaram, B. Kaymarkcalan, Dynamic Systems on Measure Chains, Kluwer Academic Publishers, Dordrecht, 1996.

[15] M. B. Mesmouli, A. Ardjouni, A. Djoudi, Existence and stability of periodic solutions for nonlinear neutral differential equations with variable delay using fixed point technique, Acta Univ. Palacki. Olomuc., Fac. rer. nat., Mathematica 54 (1) (2015), 95-108.

[16] M. B. Mesmouli, A. Ardjouni and A. Djoudi, Study of periodic and nonnegative periodic solutions of nonlinear neutral functional differential equations via fixed points, Acta Univ. Sapientiae, Mathematica, 8 (2) (2016), 255-270.

[17] D. R. Smart, Fixed point theorems, Cambridge Tracts in Mathematics, No. 66. Cambridge University Press, London-New York, 1974 . 